\title{
EFFECTS OF END-RANGE MOBILIZATION TECHNIQUE ON PAIN AND DISABILITY IN PATIENTS WITH ADHESIVE CAPSULITIS-A QUASI- EXPERIMENTAL STUDY
}

\section{ABSTRACT}

\section{BACKGROUND AND AIMS}

Studies have revealed that end-range mobilization techniques are found to be efficient in providing high and low-grade thrusts thereby enhance cellular elongation, decreases soft tissue edema and localized inflammatory changes in the affected individual. Therefore, this study is aimed to determine the effects of end-range mobilization on patients with Adhesive Capsulitis.

\section{METHODOLOGY}

This quasi-experimental study was conducted on 40 patients of 40-70 years of age with adhesive capsulitis. Endrange mobilization was performed in $2-3$ sets of $10-15$ repetitions in Grade 3 or 4 in varying plane and degree of rotation for 2 days/week for 4 weeks for the duration of 30 minutes while the Shoulder Pain and Disability Index (SPADI ) was used to assess pre and post outcomes.

\section{RESULTS}

A total number of 40 adhesive capsulitis patients included in this study with a mean age of $51.2 \pm 10.6$ consisted of 19 males and 21 females. The overall SPADI scores were significantly decreased to $66.45 \pm 8.8$ from $73.99 \pm 9.1$ postintervention of 4-weeks inclusive the marked reduction in pain and disability scores respectively

\section{CONCLUSION}

It was concluded that the end-range mobilization technique was effective to reduce pain and disability, subsequently SPADI score pre and post 4-weeks of intervention in adhesive capsulitis patients. Further trials should be conducted in the future taking large samples and varied techniques into account for the identification of optimal strategy in improving health-related outcomes of patients with shoulder joint pathologies.

\section{KEYWORDS}

AC joint, Pain, Disability, Range of Motion, Movement, Musculoskeletal
Habib Ahmed Qadri

Manager Physiotherapist

Patel Hospital Karachi

habibqadri456@gmail.com
[Qadri HA. Effects of End-Range Mobilization Technique on Pain and Disability In Patients With Adhesive Capsulitis-A Quasi-Experimental Study.Pak.j.rehabil.2021;10(1):4250]

10.36283/pjr.zu.10.1/007 


\section{INTRODUCTION}

Frozen Shoulder referred to as Adhesive Capsulitis or inflamed shoulder is a common musculoskeletal disorder characterized by pain, joint stiffness, and reduced range of motion of the shoulder joint. Pain is usually felt more at site of Deltoid insertion. Pain starts gradually with progressive loss of functional movement in capsular pattern. This is a devastating condition leading to disturbed quality of sleep and impaired quality of life due to limitation in performing activities of daily life. Several studies have shown that the condition leads to mobility deficits not only in the Glenohumeral Joint (GHJ) but also in the Scapulothoracic articulation thereby caused irregular GHJ rhythm, increased anterior scapular tilt with outward rotation, and earlier scapular upward rotation ${ }^{1}$. Frozen Shoulder is mainly idiopathic with an increased occurrence in patients with diabetes mellitus and hypothyroidism with an incidence rate of $2-5 \%$, particularly in women. Despite these factors, Adhesive Capsulitis incidence is continued to rise due to change in comorbidities and lifestyle ${ }^{2,3,4}$. Several studies have shown that more than $50 \%$ of patients with Adhesive Capsulitis suffered from chronic symptoms even after years of management that subsequently leads to decreased quality of life ${ }^{5}$. Moreover, noticeable deficits that are pain and disability were found to be common with thickening and contraction of synovial tissue in chronic conditions ${ }^{6}$. Furthermore, the number of patients with Adhesive Capsulitis may have experienced intense pain, difficulty in performing activities of daily life, and loss of motion ${ }^{7}$. Therefore, these symptoms must be managed at the earliest period of rehabilitation to ensure complete recovery and improved quality of life.

There has been a widespread myth that Frozen Shoulder may heal spontaneously however; limited evidence has been found in this regard. Moreover, around $40 \%$ of patients may have chronic symptoms with $7-15 \%$ degree of permanent loss of movement ${ }^{8}$. The condition can be improved with nonoperative and operative management strategies. Most of the time the pathology can be cured in the primary setting with the help of multimodal physical therapy treatment approaches. Several studies have proved the efficacy of physical therapy exercises, pain management modalities, mirror therapy, and manual mobilization in Adhesive Capsulitis management that are found to be appropriate to enhance mobility in patients with capsular inflammation thereby increasing ROM and decrease pain. Single and multiple dosages of Intraarticular corticosteroid therapy administration with or without mobilization techniques and home exercises have been investigated in multiples studies and promising results in terms of pain and improved ranges have been achieved clinically ${ }^{9}$. Although, various mobilization approaches along with home exercise, passive stretching have shown certain distinctions among dorsal and ventral capsular lengthening techniques with high or low-velocity thrusts ${ }^{10}$. However, these techniques are least investigated in patients with Adhesive Capsulitis and yet the protocol has not been generalized either. Moreover, studies have revealed that the end-range mobilization techniques are found to be efficient in providing high and low-grade thrusts thereby enhance cellular elongation, decreases soft tissue edema and localized inflammatory changes in the affected individuals ${ }^{11}$. However, due to varying participant characteristics, occupation, nature of pain, the stage of the disease, exercise tolerance, capacity, adherence to the treatment attitudes or mobilization, and exercise time number of approaches may produce varying significant effects ${ }^{12}$, 
hence the current study is aimed to analyze the effects of end-range mobilization on shoulder function and pain in patients with Adhesive Capsulitis.

\section{METHODOLOGY}

Study Setting: The study was conducted at the Outpatient department of Physical therapy in one of the tertiary care setting in Karachi.

Target Population: Diagnosed cases of adhesive capsulitis.

Study Design: Quasi-experimental study was conducted in the period of 6 to 8 months.

Sampling Technique and size: NonProbability Purposive Sampling Technique, 40 participants were recruited for the study.

\section{Sample Selection} Inclusion Criteria

- Both male and female patients aged 40-70 years with diagnosed adhesive capsulitis and stiff painful shoulder since 3 to 4 months ${ }^{13}$.

\section{Exclusion Criteria}

- $\quad$ Shoulder stiffness due to severe trauma, post-fracture immobilization, and surgical release of capsules.

- Patients taking oral or intraarticular steroids ${ }^{13}$.

Data Collection Tool: Data was collected at baseline and the end of treatment procedure through the Shoulder Pain and Disability Index (SPADI), a selfadministered questionnaire comprised of two dimensions i.e. pain and functional activities respectively.

The dimension of pain consisted of 5 questions regarding the severity of pain from no pain to worst imaginable pain (0-10). Functional activities dimension constitutes of total 8 questions regarding the difficulty in performing tasks from no difficulty to so difficult that it required help (0-10). SPADI required 5-10 minutes to administer while both the dimensions were assessed on $100 \%$ scores, evaluated an average score from it.

Data Collection Procedure: Data was collected from a tertiary care hospital in Karachi. Participants were recruited according to the inclusion criteria. Before the intervention, the therapist assessed the patient at baseline on SPADI and explained the protocol to ensure a better understanding of the treatment. After the completion of the treatment, pain and disability were re-assessed on a similar outcome measure. All outcome measures assessments and treatments were provided by the trained physical therapist.

Treatment

Protocol-Maitland Mobilization Technique

In the beginning, the physical therapist evaluated Pain intensity and the range of motion of the patient in all directions to gain details regarding the end-range location as well the end-feel of the GHJ respectively. After the observation, a few minutes of warm-up session consisting of rhythmic mid-range mobilizations in a supine position with the patient were performed. While maintaining this position, 10 to 15 repetitions of intense mobilization in Grade 3 or 4 were given varying the elevation plane and degree of rotation. Afterward, Maitland mobilization was performed in 2-3 sets of $10-15$ repetitions in Grade 3 or 4 in varying plane and degree of rotation. This intervention was given for 2 days/week for 4 weeks for 30 minutes of duration. The technique is described as follows:

\section{Anterior Glide}

The glide is performed on the patient in a supine lying position at the edge of the plinth, shoulder, and elbow abducted to $90 \circ$, using an anterior force by mobilizing hand to the humeral head while stabilizing the hand whereas gentle traction is applied to improve shoulder extension and external rotation. 


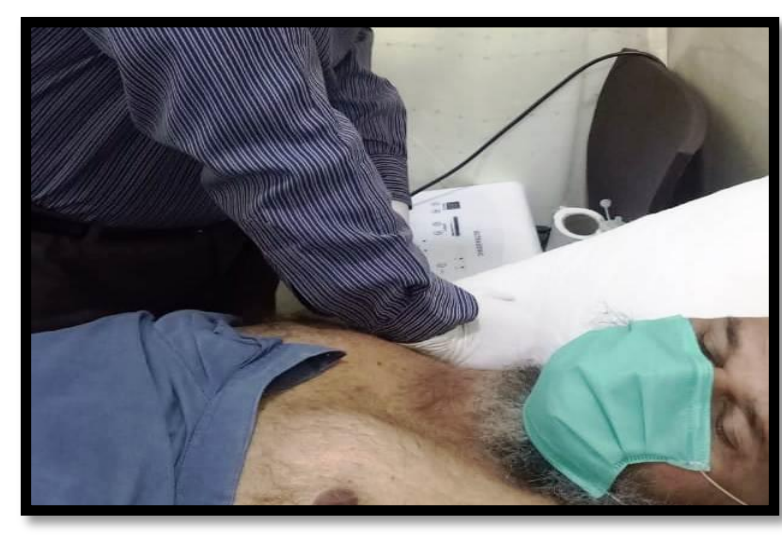

\section{Posterior Glide}

It is performed on the patient in supine at the side of the plinth with shoulder and scapula stabilized by the towel roll, maintaining an abduction of $45^{\circ}$ with the slightly flexed elbow by mobilizing hand

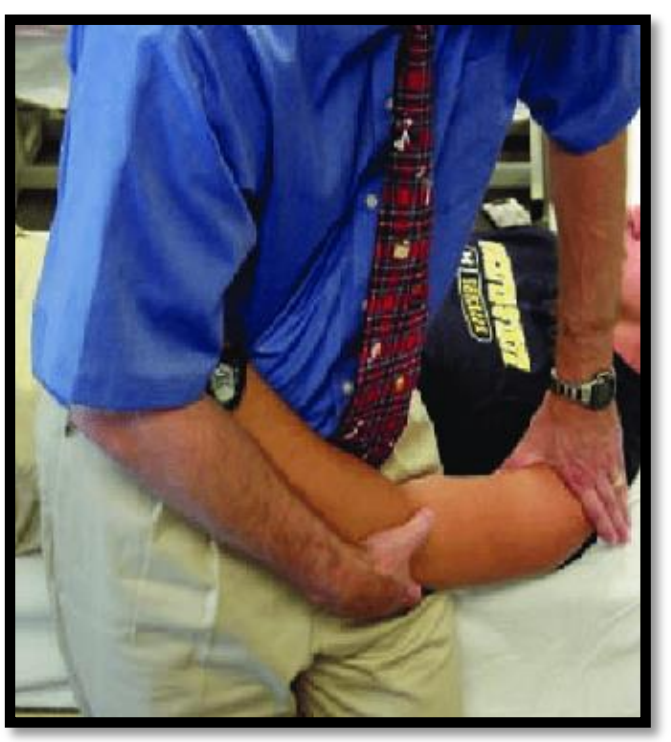

to the humeral head, posterior force is applied while stabilizing hand applies gentle traction to regain internal rotation and shoulder flexion.

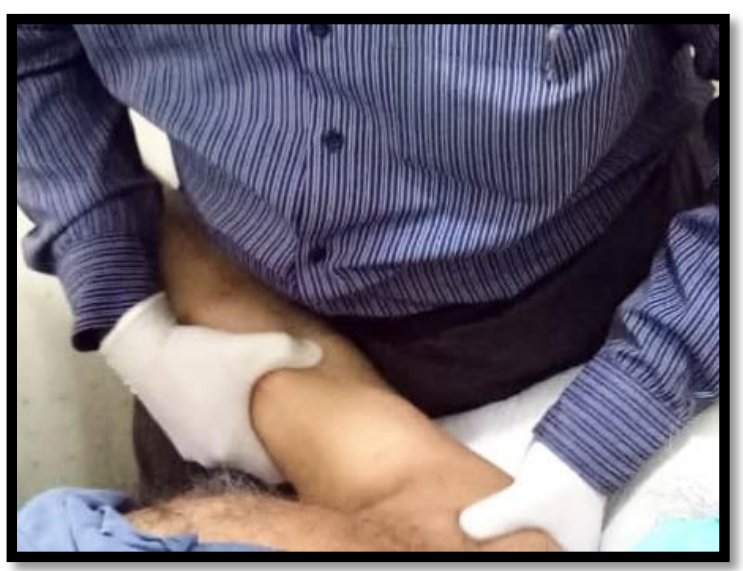

Inferior Glide
The inferior glide is applied on a patient in supine lying with the shoulder at $30^{\circ}$ to $45^{\circ}$ degrees abduction with the arm stabilizing hand, supports scapula in the axilla, mobilizing hand grasps distal humerus so that the inferior force is applied to keep the scapula secure to enhance abduction and flexion of the shoulder.

After the performance of glides, the patient was asked to perform ROM exercises consisting of the following strategies:

\section{Shoulder Pulley}

The patient was asked to be seated on a chair while holding a skipping rope, swing it up and down over an iron beam for 5 to 10 minutes to facilitate shoulder flexion and extension.

\section{Finger Ladder}

The patient was asked to position his/her affected hands over the ladder at a low angle using fingers to upward climb slowly until it reaches the top then slowly back to the starting point. The exercise was asked to be repeated 5 to 10 times.

\section{Pendulum}

The patient was asked to bend forward on a table/bench with sound forearm supported while shoulder relaxed, and gently swings affected side arm forwards and backward before experiencing a mild to moderate stretch. The patient was asked to perform these exercises with 5 to 10 repetitions in a pain-free range.

Data Analysis Strategy: Data was entered and analyzed using SPSS (Statistical Package for Social Sciences) version 20. The demographic characteristics of the participants were represented through frequency, mean and standard deviations whereas paired T-test was applied to assess pre-post difference within the group while $p<0.05$ was considered significant. 


\section{Ethical Considerations}

The researcher provided complete information about the study to every participant before the start of the study. The information procured from the participants was kept as confidential information. Every participant had full right of withdrawing from the study at any time during the study.

\section{RESULTS}

A total number of 40 adhesive capsulitis patients were included in this study according to inclusion criteria with a mean age of $51.2 \pm 10.6$ consisted of 19 males and 21 females respectively as shown in Table-1. The probability of distribution was found to be normal as depicted in Figure-1.

\begin{tabular}{|c|c|c|}
\hline Participants & \multicolumn{2}{|c|}{$\mathrm{n}=40$} \\
\hline Age & \multicolumn{2}{|c|}{$51.2 \pm 10.6$} \\
\hline \multirow[t]{2}{*}{ Gender } & Male & $19(47.5 \%)$ \\
\hline & Female & $21(52.5 \%)$ \\
\hline \multicolumn{3}{|c|}{ \pm represents Mean and Standard Deviation } \\
\hline
\end{tabular}

The participants were assessed on SPADI pre and post 4 weeks of intervention. The paired t-test was applied based on the symmetrical normality assumption. On SPADI, pain scores were significantly decreased to $68.18 \pm 10$ from $76.45 \pm 10.7$ while disability was reduced to $64.73 \pm 8.7$ from $72.40 \pm 8.7$ respectively as shown in Table-2.

Figure-1 represents normal approximation for probability histogram

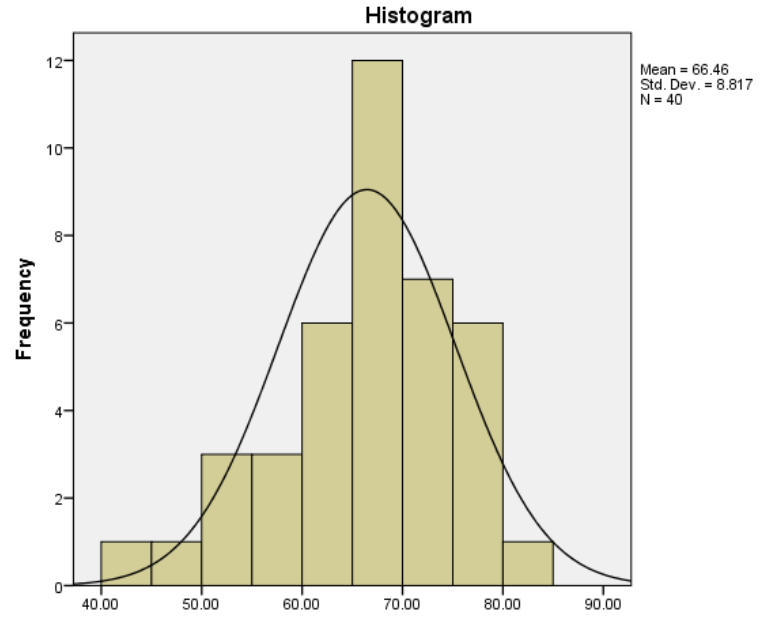

Table-2 Within the group paired t-test analysis

\begin{tabular}{|c|c|c|c|}
\hline & $\mathbf{N}$ & $\begin{array}{c}\text { Pre } \\
\text { (Mean } \pm \text { S.D.) }\end{array}$ & $\begin{array}{c}\text { Post } \\
\text { (Mean } \pm \text { S.D.) }\end{array}$ \\
\hline Pain & \multirow{2}{*}{40} & $76.45 \pm 10.7$ & $* 68.18 \pm 10$ \\
\hline Disability & & $72.40 \pm 8.7$ & *64.73 \pm 8.7 \\
\hline \multicolumn{4}{|c|}{$\begin{array}{l}\text { *represents significant reduction in SPADI scores } \\
(p<0.05)\end{array}$} \\
\hline \multicolumn{4}{|c|}{ \pm represents Mean and Standard Deviation } \\
\hline
\end{tabular}

Therefore, the overall SPADI scores were significantly decreased to $66.45 \pm 8.8$ from $73.99 \pm 9.1$ post-intervention of 4 -weeks inclusive the marked reduction in pain and disability scores respectively as shown in Figure-2.

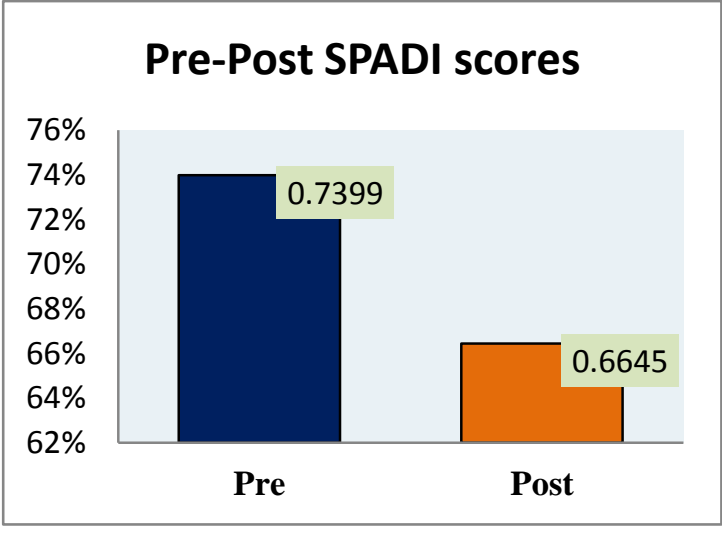

Figure-2 represents pre and post SPADI scores post-4-weeks of intervention 


\section{DISCUSSION}

This study was conducted to explore the effectiveness of the end-range mobilization technique for the treatment of adhesive capsulitis patients that involves the Maitland Technique along with ROM exercises for the duration of 4weeks. The results of our study suggest that end-range mobilization significantly improves the pain and disability of SPADI. The intervention was found to be clinically significant as it improves the shoulder range of motion and function thereby improving the quality of life in patients. Likewise, Sathe et $\mathrm{al}^{14}$ and Shamsi et $\mathrm{al}^{15}$ assessed the efficacy of the frozen shoulder by the implication of Mulligan Ultrasound Mobilization with stretching; strengthening and ROM exercises concluded that the technique was useful to reduce shoulder pain and disability hence was proven clinically significant. Besides, traditional therapy comprises gentle mobilization and passive shoulder stretching in all planes of motion or by end-range mobilization has also been suggested for the management of pain and optimal recovery of the extensibility of shoulder capsules in adhesive capsulitis patients.

However, the study conducted by Ragav and Singh $^{16}$ suggested that the Mulligan and Kaltenborn Mobilization strategies are both effective to reduce pain and improve shoulder ROM although Mulligan was found to be more successful to minimize the discomfort and join stiffness in adhesive capsulitis patients in comparison to Kaltenborn and conventional exercise programs. In particular, Almureef et al ${ }^{17}$ identified the effectiveness of mobilization technique on the quality of life of adhesive capsulitis patients by evaluating the mobilization approaches indicated that manual therapy interventions are useful to improve shoulder mobility. Furthermore, the outcome from the
Wadida et al $^{18}$ showed that for each of the three classes, levels of pain severity decreased while shoulder mobility increased. Another study reported that specific end-range mobilization and scapular mobilization have significantly higher chances to improve health-related outcomes compared to end-range mobilization alone ${ }^{19}$. However, in comparison to traditional therapy, the pain was reduced and shoulder flexion, internal rotation, and external rotation were improved in the Mobilization with Movement (MWM). On the contrary, in comparison to the phonophoresis, internal and external rotation favoring MWM were enhanced. Therefore, for optimal recovery and improved quality of life, physiotherapy is recommended in individuals with primary frozen shoulders thus therapeutic strategies are an effective choice to reduce pain and enhance the quality of life ${ }^{20,21}$. Besides, stretch mobilization combined with mobilization for posterior glide to overcome external rotation deficit is typically seen in patients with adhesive capsulitis patients. Thus, ultrasound combined with upper extremity exercises was found to be more successful ${ }^{22}$. Physical Therapists applied these techniques based on the principle of convex-on-concave joint surface movement, using an anterior glide of the humeral head to enhance external rotation, although, the intra-articular kinematic studies are still controversial therefore, the effectiveness of gliding is not well-known considering the various management strategies available for adhesive capsulitis. Moreover, the combination of several therapies may be uncertain for the varying representation of pathology among individuals therefore the outcomes to relieve pain, improve mobility and function is the utmost priority $^{23,24}$. Despite this fact, several interventions have varying results on pain, range of motion, and disability in adhesive capsulitis patients. Moreover, 
the pathogenesis of $A C$ joint is controversial; therefore, relevant information regarding the assessment and management of adhesive capsulitis is much needed to establish efficient strategies for the reliable diagnosis and treatment of shoulder joint pathologies ${ }^{25}$. To the best of the author's knowledge, this study is one of its kind to be conducted in Pakistan taking an account the effects of end-range mobilization on adhesive capsulitis patients. Furthermore, no loss of followup was reported during the implementation of the trial yet the intervention was feasible to be performed by the participants. Moreover, this study synthesizes imaging and histological research to investigate the pathophysiology associated with the primary frozen shoulder in the systematic examination. It also highlights the role of the anterior shoulder structures in the primary frozen shoulder, but there is a lack of available evidence to inform the understanding of the pathophysiology of the primary frozen shoulder disease, considered at low risk of bias. For future research direction, consensus on inclusion criteria (Codman classification criteria) will be required to encourage studies that provide comparable results. Moreover, the study has several limitations including the study design and sampling technique. Due to the ongoing Coronavirus pandemic(COVID-19), a large sample size wasn't taken because of safety precaution and social distancing as some patients might face difficulties arriving at the hospital. Moreover, the participants of this study haven't performed a home exercise program which will be beneficial and may improve the efficacy of the treatment. Similar to this study, further Randomized Controlled Trials considering large sample sizes and accessor blinding technique including control or comparative groups should be conducted on relevant primary and secondary outcome measures, analyzing various types of techniques in particular. Moreover, the extent of the research shall be extending to enhance the understanding of the disease spectrum at clearly defined stages of the disorder for better understanding and better management of this painful, debilitating common musculoskeletal disorder, unique to each point, so that identifying, managing, or describing it is no longer difficult $^{26}$.

\section{CONCLUSION}

In the current study, it was concluded that the end-range mobilization technique was effective in reducing pain and disability, subsequently SPADI score pre and post 4-weeks of intervention in adhesive capsulitis patients. Further trials should be conducted in the future taking large samples and varied techniques into account for the identification of optimal strategy in improving health-related outcomes of patients with shoulder joint pathologies.

\section{REFERENCES}

[1] Shih YF, Liao PW, Lee CS. The immediate effect of muscle release intervention on muscle activity and shoulder kinematics in patients with frozen shoulder: a cross-sectional, exploratory study. BMC musculoskeletal disorders. 2017 Dec 1;18(1):499.

[2] Cho CH, Bae KC, Kim DH. Treatment strategy for frozen shoulder. Clinics in

Orthopedic

Surgery. 2019 Sep 1;11(3):249-57.

[3] Ramirez J. Adhesive capsulitis: Diagnosis and management. American family physician. 2019 Mar 1;99(5):297-300.

[4] Park GY, Kwon DR, Kwon DG, Rim JH. Comparison of therapeutic effectiveness between shoulder distention arthrography 
with translation mobilization and distention arthrography alone in patients with frozen shoulder. Annals of rehabilitation medicine. 2018 Feb;42(1):76.

[5] Duzgun I, Turgut E, Eraslan L, Elbasan B, Oskay D, Atay OA. Which method for frozen shoulder mobilization: manual posterior capsule stretching or scapular mobilization?. Journal of Musculoskeletal \&amp; Neuronal Interactions. 2019;19(3):311.

[6] Maryam M, Kashif M, Sajjad AG, Rizwan R, Ali S, Ahmad R. Effects of posterior capsule stretch on adhesive capsulitis. The Professional Medical Journal. 2019 Aug 10;26(08):1272-7.

[7] Panchal DN, Eapen C. Effectiveness of end-range mobilization and interferential current or stretching exercise and moist heat in treatment of frozen shoulder-a randomized clinical trial.

[8] International Journal of Current Research and Review. 2015 Jul 15;7(14):21.

[9] Ryan V, Brown H, Lowe CJ, Lewis JS. The pathophysiology associated with primary

(idiopathic) frozen shoulder: A systematic review. BMC musculoskeletal disorders. 2016 Dec1;17(1):340.

[10]

itridis D, Tsikopoulos K, Bisbinas I, Papaioannidou P, Givissis P. Efficacy of pharmacological therapies for adhesive capsulitis of the shoulder: a systematic review and network metaanalysis. The American journal of sports medicine. 2019 Dec;47(14):3552-60.

[11] Brun SP. Idiopathic frozen shoulder. Australian journal of general practice. 2019 Nov;48(11):757.

[12] Lluch-Girbés E, Dueñas L, Mena-del Horno S, Luque-Suarez A, NavarroLedesma $S$, Louw A. A central nervous system-focused treatment approach for people with frozen shoulder: protocol for a randomized clinical trial. Trials. 2019 Dec 1;20(1):498.

[13] Ali SA, Khan M. Comparison for efficacy of general exercises with and without mobilization therapy for the management of adhesive capsulitis of shoulder-An interventional study. Pakistan journal of medical sciences. 2015 Nov;31(6):1372.

[14] Yeole UL, Dighe PD, Gharote GM, Panse RS, Shweta A, Pawar PA. Effectiveness of movement with mobilization in adhesive capsulitis of shoulder: Randomized controlled trial. Indian Journal of Medical Research and Pharmaceutical Sciences. 2017;4(2):1-8.

[15] Sathe S, Khurana SK, Damke U, Agrawal PV. To Compare the Effects of Maitland Mobilization with Conventional Physiotherapy in Adhesive Capsulitis. Int J Cur Res Rev| Vol.2020 Jul;12(14).

[16] Sharick Shamsi WM, Al Ghamdi AH, Shabana Khan D, Yadav A. Efficacy of mobilization in adhesive capsulitis.

[17] Ragav S, Singh A. Comparison of Effectiveness of Mulligan 'MWM'Technique versus Kaltenborn Mobilization Technique Kon Pain and End Range of Motion in

Patients with Adhesive Capsulitis of Shoulder Joint: A Randomized Controlled Trial. Journal of Exercise Science \&amp; Physiotherapy Vol. 2019;15(1).

[18] Almureef SS, Ali WM, Shamsi S, Al Zahrani MB. Effectiveness of Mobilization with Conventional Physiotherapy in Frozen Shoulder: A Systematic Review.

[19] WADIDA HE, EL SAWABEY MF, DALIA $M M$, ASHRAF NM. Effect of Mobilization with Movement versus Diclofenac 
Phonophoresis on Shoulder Adhesive Capsulitis. The Medical Journal of Cairo University. 2020 Mar 1;88(March):45-50.

[20] Sreenivasu K, Paul Daniel VK, Subramanian MB, Sajeevan $T$. Effectiveness of end range mobilization with scapular mobilization in frozen shoulder. Int Arch Integr Med. 2016;3(8):53-8.

[21] Cavalieri E, Servadio A, Berardi A, Tofani M, Galeoto G. The Effectiveness of Physiotherapy in Idiopathic or Primary Frozen Shoulder: a Systematic Review and Meta-Analysis. Muscles, Ligaments \&amp; Tendons Journal (MLTJ). 2020 Jan 1;10(1).

[22] Zavala-González J, Pavez-Baeza F, Gutiérrez-Espinoza $H$, Olguín-Huerta C. The effectiveness of joint mobilization techniques for range of motion in adult patients with primary adhesive capsulitis of the shoulder: a systematic review and meta-analysis.

[23] Biradi M, Lal RK, Sanjay P, Ahmed Z. Effects of Anterior Versus Posterior End Range Mobilizations on Shoulder Rotations Range of Motion in Adhesive Capsulitis Stage II. Indian Journal of Physiotherapy \&amp; Occupational Therapy. 2020 Apr 25;14(2):91-6.

[24] Kim Y, Lee G. Immediate Effects of Angular Joint Mobilization (a New Concept of Joint Mobilization) on Pain, Range of Motion, and Disability in a Patient with Shoulder Adhesive Capsulitis: A Case Report. The American journal of case reports. 2017;18:148.

[25] Koh KH. Corticosteroid injection for adhesive capsulitis in primary care: a systematic review of randomised clinical trials. Singapore medical journal. 2016 Dec;57(12):646.
[26] Page RS, McGee SL, Eng K, Brown G, Beattie S, Collier F, Gill SD. Adhesive capsulitis of the shoulder: protocol for the adhesive capsulitis biomarker (AdCaB) study. BMC musculoskeletal disorders. 2019 Dec 1;20(1):145.

[27] Xiao R. Comparison of Pain Relief and Functional Improvement in Landmark Vs Ultrasound- Guided Corticosteroid Injections for Adhesive Capsulitis: A Prospective Study (Doctoral dissertation) 2017 